\title{
First optical detection of the supernova remnant G 15.1-1.6
}

\author{
P. Boumis ${ }^{1}$, J. Alikakos ${ }^{1,2}$, P. E. Christopoulou ${ }^{2}$, F. Mavromatakis ${ }^{3}$, E. M. Xilouris ${ }^{1}$, and C. D. Goudis ${ }^{1,2}$ \\ 1 Institute of Astronomy \& Astrophysics, National Observatory of Athens, I. Metaxa \& V. Paulou, P. Penteli, 15236 Athens, Greece \\ e-mail: [ptb; johnal; xilouris; cgoudis]@astro.noa.gr \\ 2 Astronomical Laboratory, Department of Physics, University of Patras, 26500 Rio-Patras, Greece \\ e-mail: pechris@upatras.physics.gr \\ 3 Technological Education Institute of Crete, General Department of Applied Science, PO Box 1939, 71004 Heraklion, Crete, Greece \\ e-mail: fotis@physics.uoc.gr
}

Received 27 November 2007 / Accepted 17 January 2008

\section{ABSTRACT}

\begin{abstract}
Deep optical CCD images of the supernova remnant G 15.1-1.6 were obtained where filamentary and diffuse emission was discovered. The images, taken in the emission lines of $\mathrm{H} \alpha+[\mathrm{N} \mathrm{II}]$, [S II], and [O III], reveal filamentary and diffuse structures all around the remnant. The radio emission at $4850 \mathrm{MHz}$ in the same area is found to be strongly correlated with the brightest optical filaments. The IRAS $60 \mu$ m emission may also be correlated with the optical emission but to a lesser extent. The flux-calibrated images suggest that the optical emission originates from shock-heated gas ([S II $] / \mathrm{H} \alpha>0.4$ ), while there is a possible $\mathrm{H}$ II region ([S II ]/H $\alpha \sim 0.3$ ) contaminating the supernova remnant's emission to the east. Furthermore, deep long-slit spectra were taken at two bright filaments and also show that the emission originates from shock-heated gas. An [O III] filamentary structure is also detected farther to the west, but it lies outside the remnant's boundaries and may not be associated to it. The [O III] flux suggests shock velocities into the interstellar "clouds" $\sim 100 \mathrm{~km} \mathrm{~s}^{-1}$, while the $[\mathrm{S} \mathrm{II}] \lambda \lambda 6716 / 6731$ ratio indicates electron densities up to $\sim 250 \mathrm{~cm}^{-3}$. Finally, the $\mathrm{H} \alpha$ emission is measured to be between 2 to $7 \times 10^{-16} \mathrm{erg} \mathrm{s}^{-1} \mathrm{~cm}^{-2} \mathrm{arcsec}^{-2}$, while the lower limit to the distance is estimated at $2.2 \mathrm{kpc}$.
\end{abstract}

Key words. ISM: general - ISM: supernova remnants - ISM: individual objects: G15.1-1.6

\section{Introduction}

Supernova remants (SNRs) play an important role when studying the SN mechanism, the interstellar medium (ISM), and their interaction. Most of the SNRs have been detected in radio from their nonthermal synchrotron emission. Observations of SNRs in X-rays allow us to probe the hot gas inside the primary shock wave directly, while optical observations offer an important tool for studying of the interaction of the shock wave with dense material found in the ISM. New searches in the optical waveband continue to identify Galactic SNRs (e.g. Boumis et al. 2002, 2005; Mavromatakis et al. 2002, 2005), while in the past decade, observations in X-rays have also detected new Galactic SNRs (e.g. Seward et al. 1995; see also Green 2006, for a complete catalogue).

G 15.1-1.6 is not a well-known SNR. It was first detected by Reich et al. (1988) in the Effelsberg $2.7 \mathrm{GHz}$ survey, and the radio image was published by Reich et al. (1990). It is classified as a shell-type SNR with a spectral index of $\sim 0.8$. Its angular size is $30^{\prime} \times 24^{\prime}$, and using the brightness-to-diameter $(\Sigma-\mathrm{D})$ relationship, the distance of the remnant was calculated at $5.7 \mathrm{kpc}$ (Green 2006). Radio surveys of the surrounding area do not reveal any pulsar associated with G 15.1-1.6, while it is not detected optically in the past.

In this paper we report the optical detection of G 15.1-1.6. We present images of the remnant in the $\mathrm{H} \alpha+[\mathrm{N} \mathrm{II}],[\mathrm{S} \mathrm{II}]$, and [O III] emission lines. Deep long-slit spectra were also acquired in a number of selected areas. In Sect. 2 we present information about the observations and data reduction, while the results of the imaging and spectroscopic observations are given in Sect. 3 . In Sect. 4 we discuss the optical properties of this SNR, while we summarize the results of this work in Sect. 5.

\section{Observations}

A summary and log of our observations are given in Table 1. In the sections below, we describe the details of these observations.

\subsection{Imaging}

\subsubsection{Wide-field imagery}

G 15.1-1.6 was observed with the $0.3 \mathrm{~m}$ Schmidt-Cassegrain $(f / 3.2)$ telescope at Skinakas Observatory, Crete, Greece in June 11 and August 27, 28, and 30, 2005. The data were taken with a $1024 \times 1024$ Thomson CCD with a pixel size of $19 \mu \mathrm{m}$ resulting in a $70^{\prime} \times 70^{\prime}$ field of view and an image scale of $4^{\prime \prime}$ per pixel. The area of the remnant was observed with the $\mathrm{H} \alpha+[\mathrm{N} \mathrm{II}],[\mathrm{S} \mathrm{II}]$, and [O III] filters. The exposure time was set to $2400 \mathrm{~s}$ for each observation and to $180 \mathrm{~s}$ for the continuum red and blue filters. The continuum-subtracted images of the $\mathrm{H} \alpha+[\mathrm{N} \mathrm{II}]$ and $[\mathrm{O}$ III $]$ emission lines are shown in Figs. 1 and 2 , respectively.

The IRAF and MIDAS packages were used for the data reduction. All frames were bias-subtracted and flatfield-corrected using a series of twilight flatfields. Using the continuum images multiplied with a proper factor, we subtracted the stars in order to present the remnant. The spectrophotometric standards stars HR5501, HR7596, HR7950, HR8634, and HR9087 (Hamuy et al. 1992) were used for the absolute flux calibration. The astrometric solution for all data frames were calculated using the Hubble Space Telescope (HST) Guide Star Catalogue (Lasker et al. 1999). All the equatorial coordinates quoted in this work refer to epoch 2000 . 
Table 1. Imaging and spectral log.

\begin{tabular}{|c|c|c|c|c|c|c|}
\hline \multirow[b]{2}{*}{ Filter } & \multicolumn{4}{|c|}{ 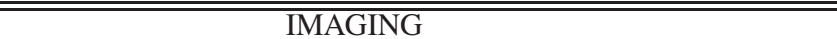 } & \multirow[b]{2}{*}{ Telescope } & \\
\hline & $\begin{array}{l}\lambda_{\mathrm{c}} \\
(\AA)\end{array}$ & $\begin{array}{l}\Delta \lambda \\
(\AA)\end{array}$ & $\begin{array}{c}\text { Total exp. time } \\
(\mathrm{sec})\end{array}$ & $\mathrm{N}^{0}$ of diff. fields & & \\
\hline $\mathrm{H} \alpha+[\mathrm{N}$ II $] 6548 \& 6583 \AA$ & 6570 & 75 & $7200(3)^{a}$ & 1 & $0.3-\mathrm{m}$ & \\
\hline [O III $] 5007 \AA$ & 5010 & 28 & $7200(3)$ & 1 & $0.3-\mathrm{m}$ & \\
\hline [S II] $6716 \& 6731 \AA$ & 6720 & 18 & $4800(2)$ & 1 & $0.3-\mathrm{m}$ & \\
\hline Cont blue & 5470 & 230 & 180 & 1 & $0.3-\mathrm{m}$ & \\
\hline Cont red & 6096 & 134 & 180 & 1 & $0.3-\mathrm{m}$ & \\
\hline [O III] $5007 \AA$ & 5010 & 28 & 2400 & 9 & $1.3-\mathrm{m}$ & \\
\hline Cont blue & 5470 & 230 & 180 & 9 & $1.3-\mathrm{m}$ & \\
\hline $\mathrm{H} \alpha+[\mathrm{N} \mathrm{II}] 6548 \& 6583 \AA$ & 6570 & 75 & 2400 & 9 & $1.3-\mathrm{m}$ & \\
\hline Cont red & 6096 & 134 & 180 & 9 & $1.3-\mathrm{m}$ & \\
\hline \multirow{3}{*}{ Area } & \multicolumn{4}{|c|}{ SPECTROSCOPY } & \multirow{3}{*}{$\begin{array}{l}\text { Aperture length }{ }^{c} \\
\quad(\operatorname{arcsec})\end{array}$} & \multirow{3}{*}{ Telescope } \\
\hline & Slit c & nters & Total exp. time & Offset $^{b}$ & & \\
\hline & $\alpha$ & $\delta$ & $(\mathrm{s})$ & $(\operatorname{arcsec})$ & & \\
\hline North (BI) & $18^{\mathrm{h}} 23^{\mathrm{m}} 44.0^{\mathrm{s}}$ & $-16^{\circ} 29^{\prime} 05^{\prime \prime}$ & $7800(2)$ & $10.6 \mathrm{~N}$ & 38.9 & $1.3-\mathrm{m}$ \\
\hline North (BII) & $18^{\mathrm{h}} 23^{\mathrm{m}} 44.0^{\mathrm{s}}$ & $-16^{\circ} 29^{\prime} 05^{\prime \prime}$ & $7800(2)$ & $96.8 \mathrm{~N}$ & 13.0 & $1.3-\mathrm{m}$ \\
\hline South-East (E1) & $18^{\mathrm{h}} 24^{\mathrm{m}} 14.6^{\mathrm{s}}$ & $-16^{\circ} 36^{\prime} 38^{\prime \prime}$ & 3900 & $123.3 \mathrm{~S}$ & 15.3 & $1.3-\mathrm{m}$ \\
\hline
\end{tabular}

${ }^{a}$ Numbers in parentheses represent the number of individual frames.

${ }^{b}$ Spatial offset from the slit center in arcsec: N(=North), S(=South).

${ }^{c}$ Aperture lengths for each area in arcsec.

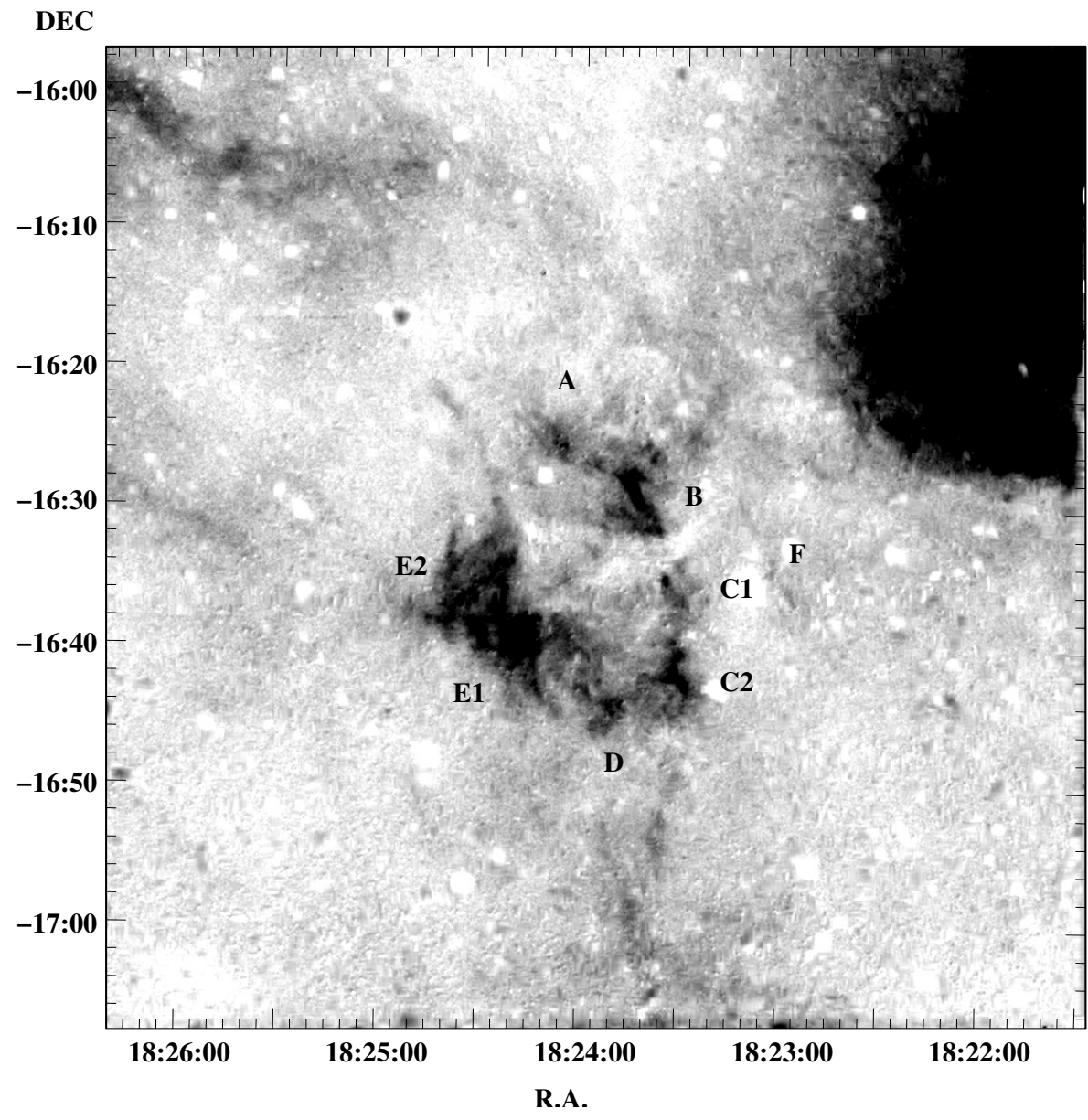

Fig. 1. The G $15.1-1.6$ in the $\mathrm{H} \alpha+[\mathrm{N}$ II] filter. Shadings run linearly from 0 to $220 \times$ $10^{-17} \mathrm{erg} \mathrm{s}^{-1} \mathrm{~cm}^{-2} \operatorname{arcsec}^{-2}$. The image has been smoothed to suppress the residuals from the imperfect continuum subtraction. The bright region in the northwest edge of the image is a known H II region (Lockman 1989).

\subsubsection{High-resolution imagery}

Optical images at higher angular resolution of G 15.1-1.6 were also obtained with the $1.3 \mathrm{~m}(f / 7.7)$ Ritchey-Cretien telescope at Skinakas Observatory in July 4-7 and 8-10, 2007, using the $\mathrm{H} \alpha+[\mathrm{N} \mathrm{III}]$ and the [O III $]$ interference filters, respectively. The detector was a $1024 \times 1024$ SITe CCD with a field of view of
$8.5 \times 8.5 \operatorname{arcmin}^{2}$ and an image scale of $0.5^{\prime \prime}$ per pixel. Nine exposures were taken through the $\mathrm{H} \alpha+[\mathrm{N} \mathrm{II}]$ and [O III] filters each of $2400 \mathrm{~s}$ and nine corresponding exposures in the continuum, each of $180 \mathrm{~s}$. During the observations, the "seeing" varied between $0.8^{\prime \prime}$ and $1.5^{\prime \prime}$, while the full width at half maximum (FWHM) of the star images was between 1.2" and 2.1". 


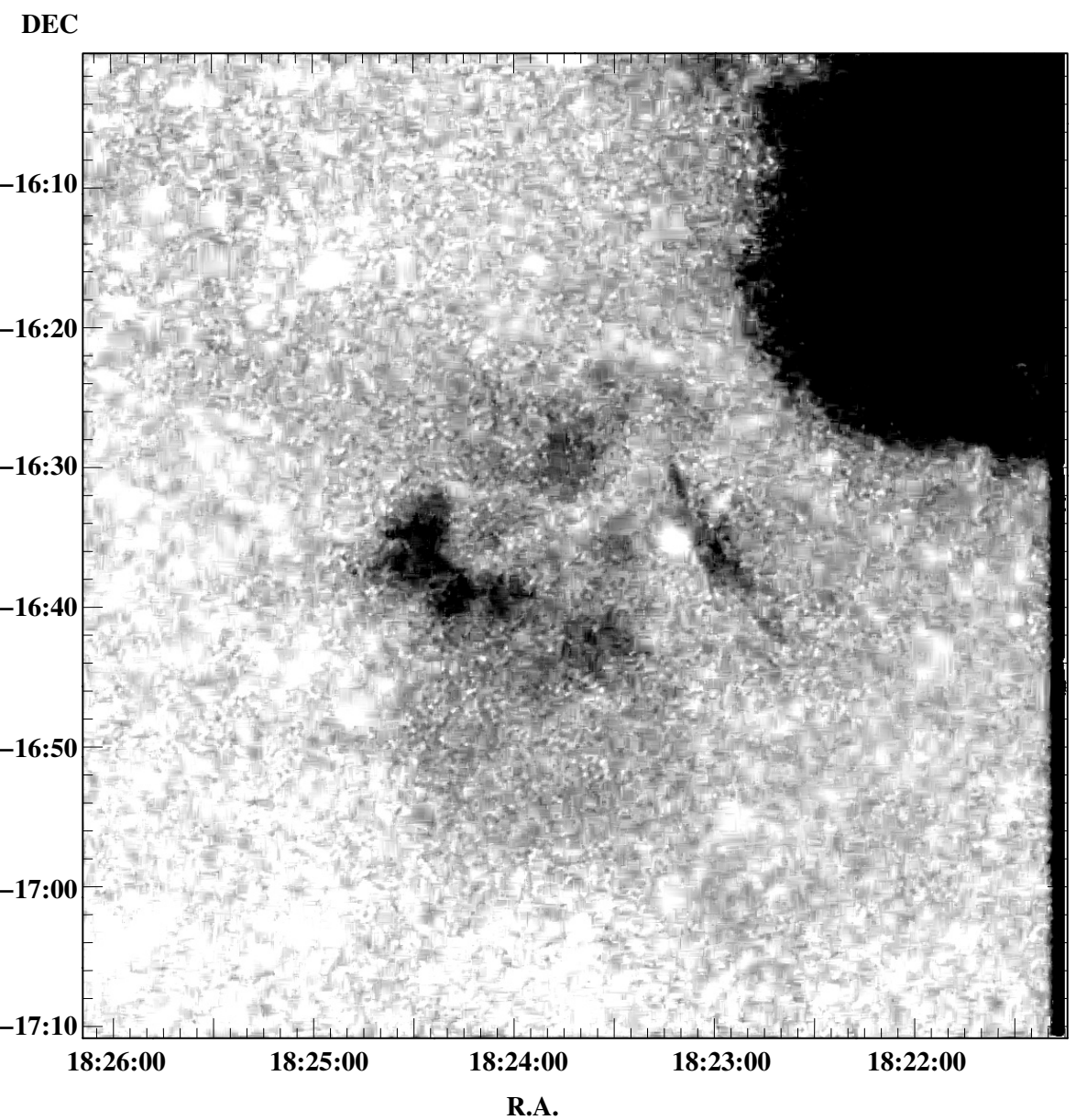

Fig. 2. The G $15.1-1.6$ in the [O III] filter. Shadings run linearly from 0 to $45 \times$ $10^{-17} \mathrm{erg} \mathrm{s}^{-1} \mathrm{~cm}^{-2} \operatorname{arcsec}^{-2}$. The image has been smoothed to suppress the residuals from the imperfect continuum subtraction.
The continuum-subtracted mosaic of the $\mathrm{H} \alpha+[\mathrm{N} \mathrm{II]}$ and [O III] images are shown in Figs. 3 and 4, respectively.

\subsection{Spectroscopy}

The $1.3 \mathrm{~m}$ Ritchey-Cretien (f/7.7) telescope at Skinakas Observatory was used to obtain low-dispersion, long-slit spectra on June 4 and 5 and September 7, 2005. The exposure time was $3900 \mathrm{~s}$, except for the north area of $\mathrm{G}$ 15.1-1.6, which was $7800 \mathrm{~s}$. The data were taken with a 1300 line $\mathrm{mm}^{-1}$ grating and a $2000 \times 800(13 \mu \mathrm{m})$ SITe CCD covering the range $4750 \AA-6815 \AA$. The spectral resolution is $\sim 8$ pixels and $\sim 11$ pixels full width at half maximum (FWHM) for the red and blue wavelengths, respectively. The airmass of the object varied between 1.6 and 1.7. The slit has a width of 7'.7 and length of 7.9 and was oriented in all cases in the south-north direction. The coordinates of the slit centers of each spectrum are given in Table 1. For the absolute calibration we used the spectrophotometric standard stars HR4468, HR5501, HR7596, HR7950, HR8634, and HR9087. The data reduction was performed with the IRAF package.

\section{Results}

\subsection{The $\mathrm{H} \alpha+[\mathrm{N} I I]$, [S II], and [O III] emission-line images}

Optical filamentary and diffuse emission was detected for the first time for this remnant with several thin and curved filaments found all around the remnant. The most interesting regions, where complex filamentary structures exist, lie in the northwest, west, and southeast. In Table 2, we present typical, average fluxes measured in several locations within the field of G 15.1-1.6. The detected [S II] emission appears more diffuse and less filamentary than in the $\mathrm{H} \alpha+[\mathrm{N} \mathrm{II}]$ image; however, its morphology is similar to the $\mathrm{H} \alpha+[\mathrm{N}$ II], so it is not shown here.

Starting from the north, a bright filament $2^{\prime}$ long (named A in Fig. 1) is present with its center approximately at $\alpha \simeq 18^{\mathrm{h}} 24^{\mathrm{m}} 04^{\mathrm{s}}$ and $\delta \simeq-16^{\circ} 25^{\prime} 47^{\prime \prime}$. This filament lies a few arcminutes to the northeast of the very bright $4^{\prime}$ filamentary structure (named B) which is between $\alpha \simeq 18^{\mathrm{h}} 23^{\mathrm{m}} 49^{\mathrm{s}}, \delta \simeq-16^{\circ} 27^{\prime} 26^{\prime \prime}$ and $\alpha \simeq$ $18^{\mathrm{h}} 23^{\mathrm{m}} 36^{\mathrm{s}}, \delta \simeq-16^{\circ} 30^{\prime} 55^{\prime \prime}$. In particular, there is a very bright filament $3^{\prime}$ long and $1.2^{\prime}$ wide with $\sim 1^{\prime}$ diffuse emission in its south. Southwest of this filament a fainter one (C1) appears at $\alpha \simeq 18^{\mathrm{h}} 23^{\mathrm{m}} 34^{\mathrm{s}}, \delta \simeq-16^{\circ} 35^{\prime} 08^{\prime \prime}$, which is up to $1^{\prime}$ long separated by a $2.5^{\prime}$ gap with area B. This gap is due to a dark region in the area (probably created by dust - supported also by the IRAS map) preventing the detection of optical emission from the SNR. Farther to the south, there is a prominent bright structure that also appears strong and is designated as filament $\mathrm{C} 2$. This structure $\left(\sim 3^{\prime}\right.$ long, $\sim 40^{\prime \prime}$ wide) lies at $\alpha \simeq 18^{\mathrm{h}} 23^{\mathrm{m}} 30^{\mathrm{s}}$, $\delta \simeq-16^{\circ} 41^{\prime} 56^{\prime \prime}$. To the south, there is fainter $1^{\prime}$ long emission (named D), which has strong [S II] emission like the filament in area $\mathrm{A}([\mathrm{S} \mathrm{II}] / \mathrm{H} \alpha \sim 0.7)$. The east structure consists of two main parts; a very bright one that covers an area of $\sim 4 \times 4 \mathrm{arcmin}^{2}$, centered at $\alpha \simeq 18^{\mathrm{h}} 24^{\mathrm{m}} 17^{\mathrm{s}}, \delta \simeq-16^{\circ} 39^{\prime} 36^{\prime \prime}$ (E1), and a more complex but less bright one between $\alpha \simeq 18^{\mathrm{h}} 24^{\mathrm{m}} 38^{\mathrm{s}}$, $\delta \simeq-16^{\circ} 31^{\prime} 28^{\prime \prime}$, and $\alpha \simeq 18^{\mathrm{h}} 24^{\mathrm{m}} 20^{\mathrm{s}}, \delta \simeq-16^{\circ} 37^{\prime} 51^{\prime \prime}$ (E2). Diffuse emission is also present close to the filamentary structures, as well as the center of the SNR. All filamentary structures have the same curvature, which supports their belonging to 


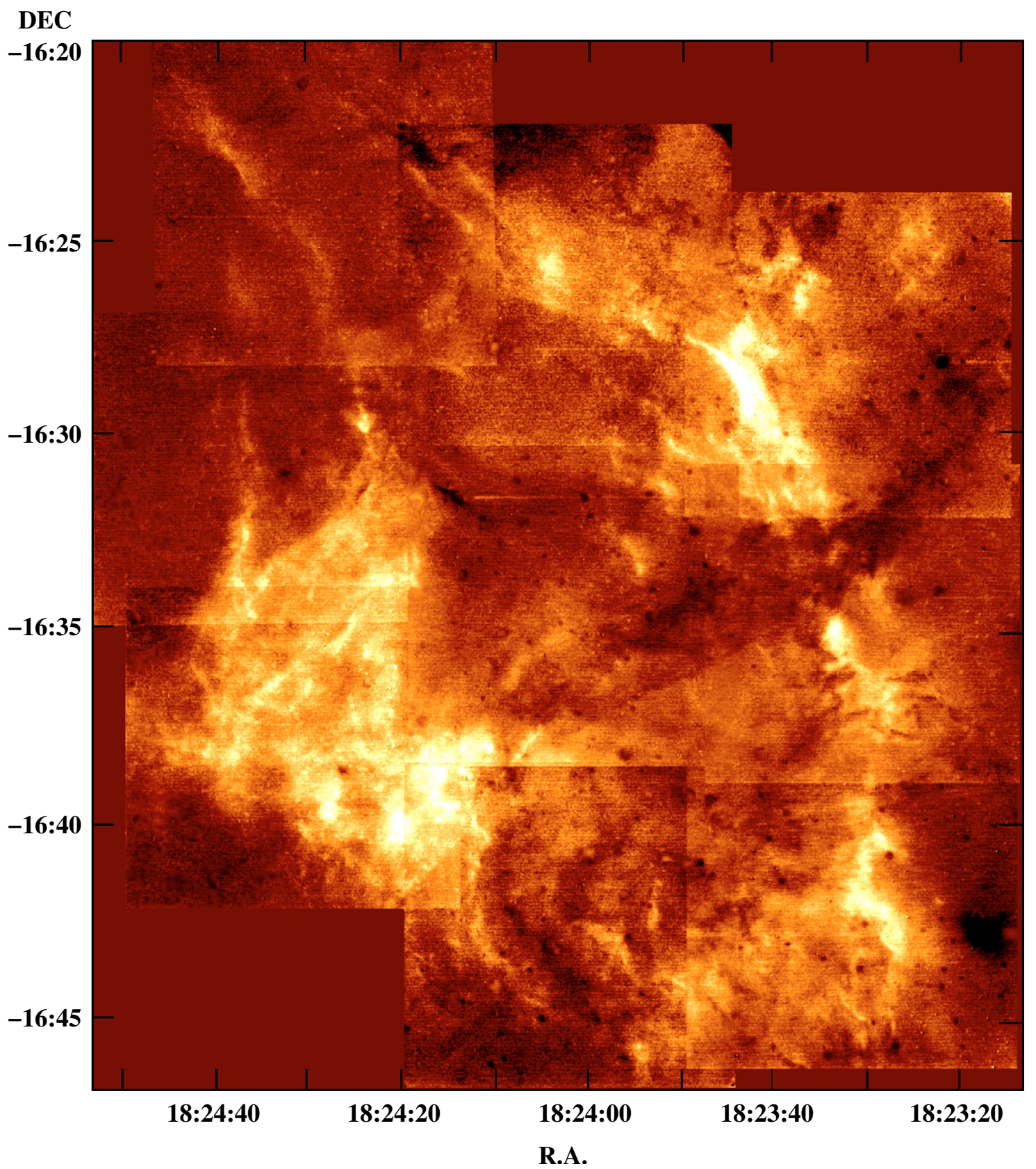

Fig. 3. The continuum-subtracted mosaic of G 15.1-1.6 taken with the 1.3-m telescope in the light of $\mathrm{H} \alpha+[\mathrm{N}$ II]. The image has been smoothed to suppress the residuals from the imperfect continuum subtraction.

G 15.1-1.6. It is interesting to note two very thin long filaments to the north of E2 extending for $\sim 10^{\prime}$ and joined to a $2.5^{\prime}$ single filament. Similar filaments also apear to the northeast of area A. Both seem to follow the infrared emission but the low resolution of the latter does not allow a detailed investigation.

The detected [O III] emission (Figs. 2, 4) appears less filamentary and more diffuse than in the $\mathrm{H} \alpha+[\mathrm{N}$ II] image. Typical [O III] fluxes are listed in Table 2. Significant differences between the $\mathrm{H} \alpha+[\mathrm{NII}]$ and $[\mathrm{O} \mathrm{III}]$ images are present for many of the filaments. In particular, to the north, west, and south (A, B, C, and D), in contrast to the bright filaments found in $\mathrm{H} \alpha+[\mathrm{N}$ II] , the [O III] displays a different morphology with much fainter diffuse emission. Only within area $\mathrm{B}$, there is a bright [O III] filament centered at $\alpha \simeq 18^{\mathrm{h}} 23^{\mathrm{m}} 43^{\mathrm{s}}, \delta \simeq-16^{\circ} 28^{\prime} 30^{\prime \prime}$, which lies exactly at the same position with the bright one in $\mathrm{H} \alpha+[\mathrm{NII}]$. Farther to the west, there is a bright [O III] filament (named F) splits to a very thin one $\left(\sim 30^{\prime \prime}\right.$ wide) at $\alpha \simeq$ $18^{\mathrm{h}} 23^{\mathrm{m}} 10^{\mathrm{s}}, \delta \simeq-16^{\circ} 31^{\prime} 25^{\prime \prime}$ and a wider complex structure at $\alpha \simeq 18^{\mathrm{h}} 22^{\mathrm{m}} 59^{\mathrm{s}}, \delta \simeq-16^{\circ} 35^{\prime} 49^{\prime \prime}$. This filament does not have an $\mathrm{H} \alpha+[\mathrm{N} \mathrm{II}]$ counterpart, and only very faint diffuse emission is found at the same location. However, it is not correlated with the $4850 \mathrm{MHz}$ radio map (Fig. 6) of G 15.1-1.6 and probably does not belong to the remnant. On the other hand, the bright $\mathrm{H} \alpha+[\mathrm{N} \mathrm{II}]$ emission found to the east (area E) also appears bright but less filamentary in [O III]. Finally, a similar situation appears both in $[\mathrm{O}$ III $]$ and $\mathrm{H} \alpha+[\mathrm{N} \mathrm{II}]$ in the areas where weak and diffuse emission is found.

All images being flux-calibrated provides a first indication of the nature of the observed emission. An examination of the diagnostic ratio $[\mathrm{S} \mathrm{II}] / \mathrm{H} \alpha$ shows that the emission from the brightest parts of the remnant originates from shock-heated gas, since we estimate ratios $[\mathrm{S} \mathrm{II}] / \mathrm{H} \alpha$ of $0.4-0.6$, which agree with our 
DEC

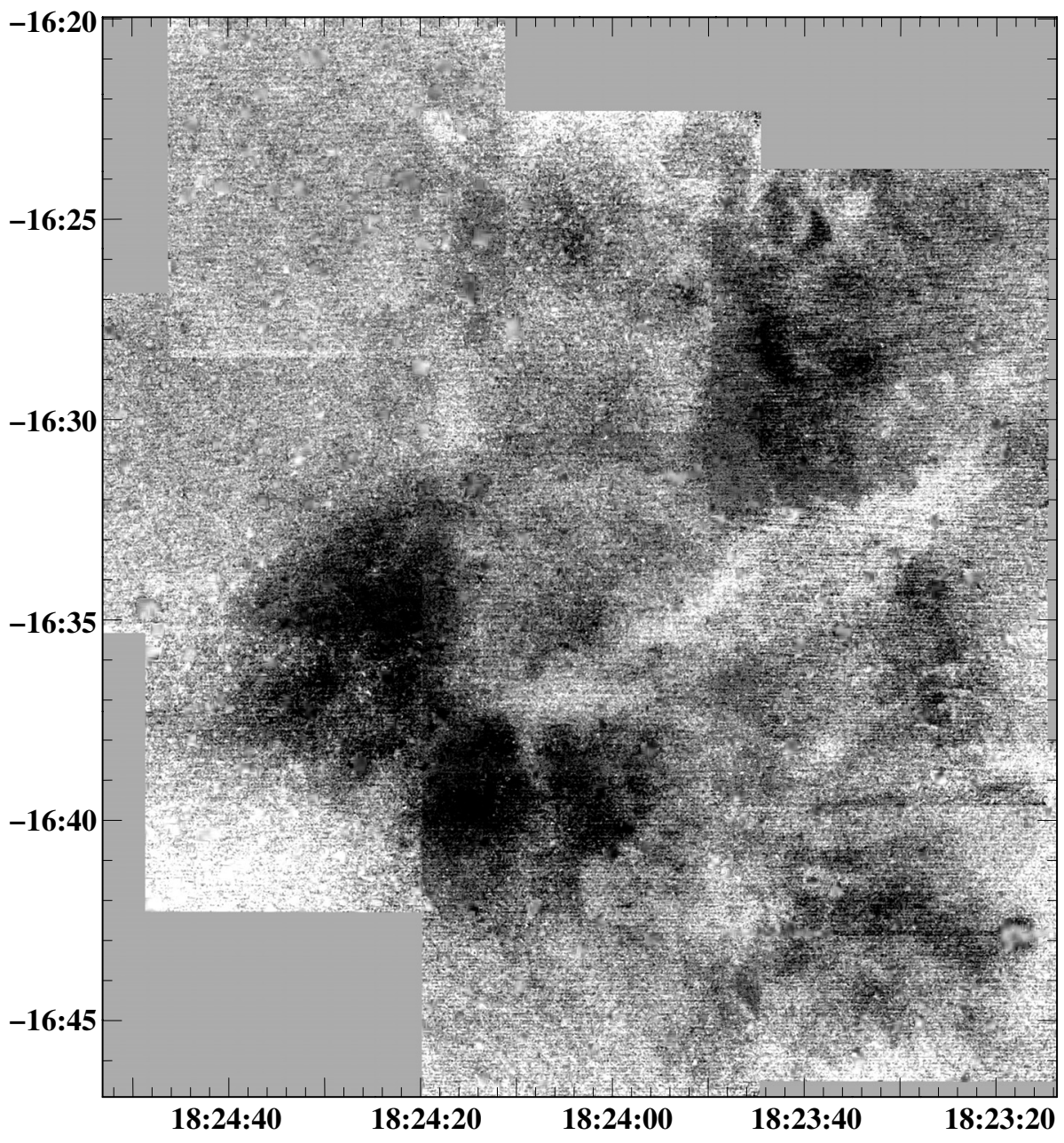

R.A.
Fig. 4. The continuum-subtracted mosaic of G 15.1-1.6 taken with the 1.3-m telescope in the light of [O III]. The image has been smoothed to suppress the residuals from the imperfect continuum subtraction. spectral measurements (Sect. 3.2). The northern and southern areas (A and D) show [S II]/H $\alpha \sim 0.7$. A photoionization mechanism may be producing the emission in the southeastern region since the ratio $[\mathrm{S} \mathrm{II}] / \mathrm{H} \alpha$ is $\sim 0.3$. The possibility that an $\mathrm{H} \mathrm{II} \mathrm{emis-}$ sion contaminates the remnant's emission to the east (E) cannot be ruled out since, for some of the areas close to both the filament and to the central region of the remnant, we estimate [S II $] / \mathrm{H} \alpha \sim 0.3-0.4$ which also agrees with our spectra.

Assuming that all the bright filaments belong to the remnant, their geometry allows us to define its diameter approximately. In particular, the eastern border of the remnant is defined by the outer filament at $\alpha \simeq 18^{\mathrm{h}} 24^{\mathrm{m}} 40.0^{\mathrm{s}}$, the western border at $\alpha \simeq 18^{\mathrm{h}} 23^{\mathrm{m}} 25.0^{\mathrm{s}}$, the northern border at $\delta \simeq-16^{\circ} 24^{\prime}$, and the southern border at $\delta \simeq-16^{\circ} 46^{\prime}$. Then a diameter of $30^{\prime} \times 24^{\prime}$ can be derived with its center at $\alpha \simeq 18^{\mathrm{h}} 24^{\mathrm{m}} 00^{\mathrm{s}}, \delta \simeq-16^{\circ} 35^{\prime} 20^{\prime \prime}$. The optically derived angular size is identical to what is quoted in Green's catalogue (Green 2006).

\subsection{The optical spectra from $G 15.1-1.6$}

The deep low-resolution spectra were taken on the relatively bright optical filaments at two different locations (Table 1). Table 3 quotes the relative line fluxes taken from the above locations (designated Area B and E). In particular, we extracted two different apertures (BI and BII) in Area B, along the slit that are free of field stars and include enough line emission to allow an accurate determination of the observed lines. The background extraction apertures were selected towards the northern and southern ends of each slit depending on the filament's position within the slit. The measured line fluxes indicate emission from shock-heated gas, since $[\mathrm{S} \mathrm{II}] / \mathrm{H} \alpha \simeq 0.5$. Furthermore, the $[\mathrm{N} \mathrm{II}] / \mathrm{H} \alpha$ ratio, which takes values between 0.63 and 1.07 (see Table 3, falls well inside the range expected for an SNR (Fesen et al. 1985). The signal-to-noise ratios do not include calibration errors, which are less than 10 percent. Typical spectra from the north (BI) and south (E1) are shown in Fig. 5.

The absolute $\mathrm{H} \alpha$ flux covers a range of values from 2 to $7 \times 10^{-16} \mathrm{erg} \mathrm{s}^{-1} \mathrm{~cm}^{-2} \operatorname{arcsec}^{-2}$. The [S II] $\lambda \lambda$ 6716/6731 ratio that was calculated between 1.3 and 1.4 , indicates electron densities between 40 and $150 \mathrm{~cm}^{-3}$ (Osterbrock \& Ferland 2006). However, taking the statistical errors on the sulfur lines into account, we calculate that electron densities up to $250 \mathrm{~cm}^{-3}$ are allowed (Shaw \& Dufour 1995). Measurements for the [O III]/ $\mathrm{H} \beta$ ratio result in values less than 6 . Theoretical models of Cox \& Raymond (1985) and Hartigan et al. (1987) suggest that this value is $\sim 6$ for shocks with complete recombination zones, while this limit is exceeded in case of shock with incomplete recombination zones (Raymond et al. 1988). Our measured values suggest that shocks with complete recombination zones are present. Therefore, according to our measurements and the theoretical models above, the estimated shock velocities are $\sim 100 \mathrm{~km} \mathrm{~s}^{-1}$. 


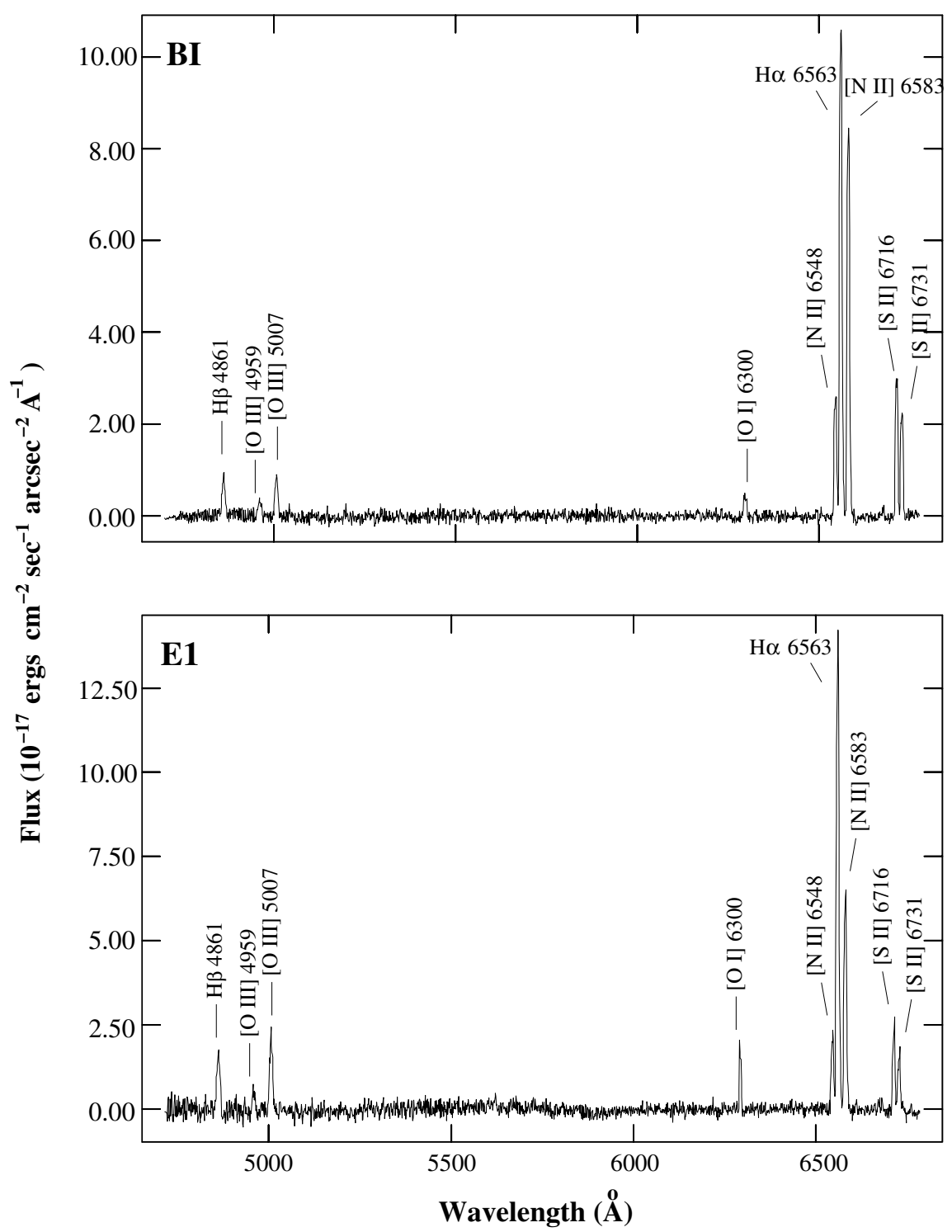

Fig. 5. Typical long-slit spectra.

\subsection{Observations at other wavelengths}

The optical emission matches the radio emission of G 15.1-1.6 very well at $4850 \mathrm{MHz}$, suggesting their correlation (Fig. 6). The observed filaments are located close to the outer edge of the radio contours but the low resolution of the radio images does not allow us to determine the relative position of the filament with respect to the shock front. To explore how the optical emission correlates with the infrared emission, we examined IRAS images at $60 \mu \mathrm{m}$ of the same area. Figure 6 shows a deep greyscale representation of the optical emission $(\mathrm{H} \alpha+[\mathrm{N}$ II $])$ with overlapping contours of the infrared emission $(60 \mu \mathrm{m})$. Although the low-resolution IRAS map does not permit a detailed comparison with the optical image, there is a clear enhancement of infrared emission in the area where the optical emission of the SNR is detected. The infrared emission follows the morphology of the SNR closely, but it also fills the central area that shows no optical emission. We also examined the ROSAT All-sky survey data, but no significant X-ray emission was detected.

\section{Discussion}

The supernova remnant G 15.1-1.6 shows up as an almost complete shell in the radio band without any X-ray emission detected so far. The absence of soft X-ray emission may indicate a low shock temperature and/or a low density of the local interstellar medium. The $\mathrm{H} \alpha+[\mathrm{NII}]$ image describes the newly detected structures best. Both [S II] and [O III] emission is also detected and generally appears less filamentary and more diffuse than in the $\mathrm{H} \alpha+[\mathrm{N} \mathrm{II}]$ image with their position and shape in agreement with that of the $\mathrm{H} \alpha+[\mathrm{N} \mathrm{II}]$. The [O III] emission does not seem to be bounded by $\mathrm{H} \alpha+[\mathrm{N}$ II $]$ emission. An explanation is given in Blair et al. (2005), indicating that the shock emission from the nascent radiative region is strong enough to fully ionize the local preshock gas. The presence of [O I] $6300 \AA$ line emission is also consistent with the emission being shock material. Both the calibrated images and the long-slit spectra suggest that the detected emission results from shock-heated gas since the $[\mathrm{S} \mathrm{II}] / \mathrm{H} \alpha$ ratio exceeds the empirical SNR criterion value of 0.4-0.5, while the measured $[\mathrm{N} \mathrm{II}] / \mathrm{H} \alpha$ ratio also confirms this result. Note that the possible $\mathrm{H}$ II region found in the low ionization images shows an [S II] $/ \mathrm{H} \alpha$ ratio of $\sim 0.33$. The eastern filament lies very close to this H II region. The morphological differences between the low and medium ionization lines provide evidence of significant inhomogeneities and density variations in the ambient medium. Hester et al. (1987) suggested that the presence of these inhomogeneities and density variations would mainly affect the 


\section{DEC}

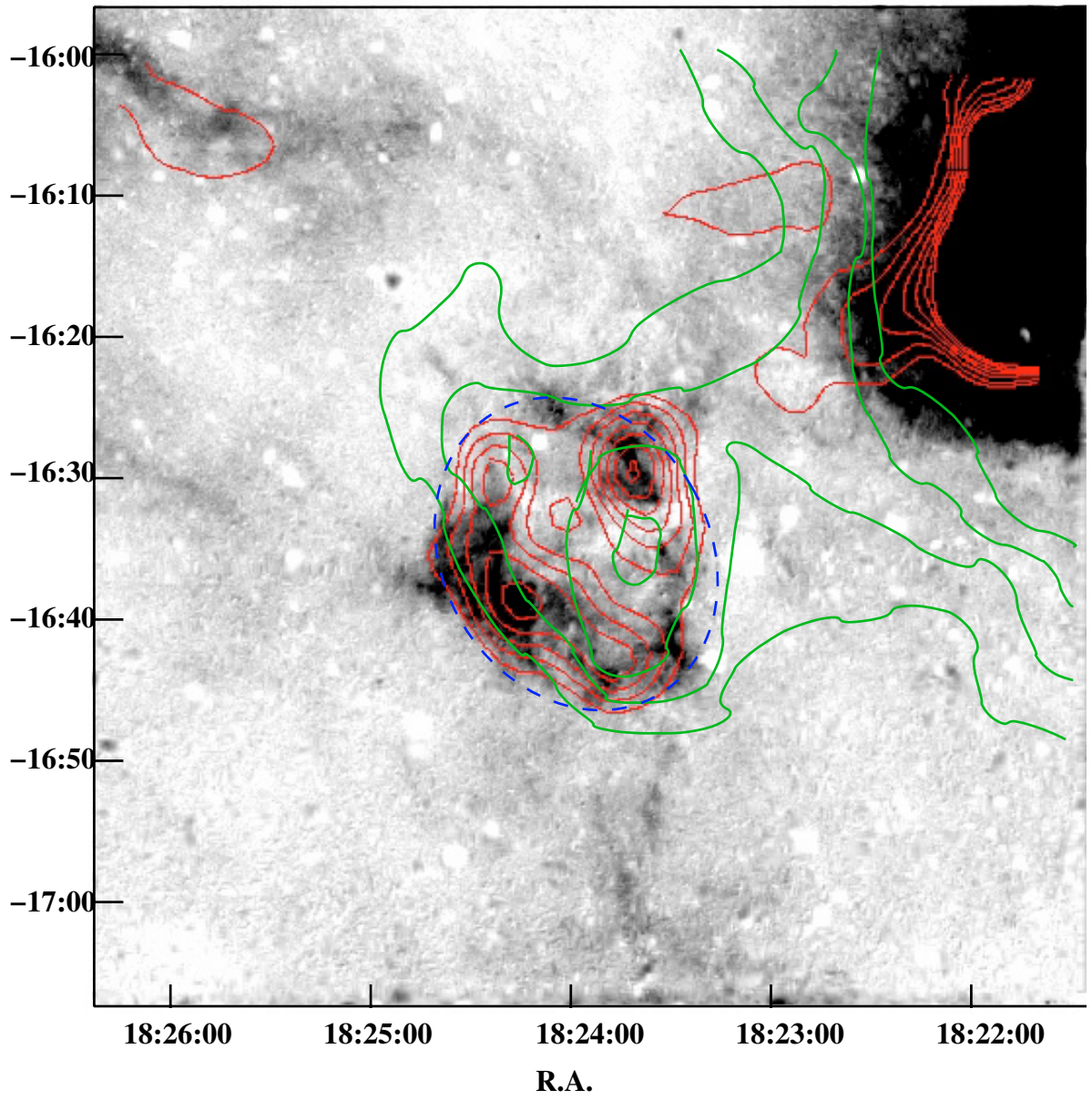

Fig. 6. The correlation between the $\mathrm{H} \alpha+[\mathrm{NII}]$ emission, and the radio emission at $4850 \mathrm{MHz}$ (solid red line), the infrared emission at IRAS $60 \mu \mathrm{m}$ (solid green line). The $4850 \mathrm{MHz}$ radio contours scale linearly from $3.54 \times 10^{-2} \mathrm{Jy} /$ beam, to $0.3 \mathrm{Jy} /$ beam while the $60 \mu \mathrm{m}$ contours are at the level of 170, 200, 230, and $260 \mathrm{MJy} / \mathrm{sr}$. The ellipse (dash blue line) defines the SNR's boundary approximately.
Table 2. Typically measured fluxes over the brightest filaments.

\begin{tabular}{lllllll}
\hline \hline & $\mathrm{A}$ & $\mathrm{B}$ & $\mathrm{C}$ & $\mathrm{D}$ & $\mathrm{E}$ & $\mathrm{F}^{a}$ \\
\hline $\mathrm{H} \alpha+[\mathrm{N}$ II $]$ & 86 & 156 & 111 & 80 & 152 & 24 \\
{$[\mathrm{~S} \mathrm{II}]$} & 30 & 38 & 30 & 28 & 37 & $<5^{b}$ \\
{$[\mathrm{O}$ III $]$} & 12 & 5 & 20 & 9 & 16 & 21 \\
\hline
\end{tabular}

Fluxes in units of $10^{-17} \mathrm{erg} \mathrm{s}^{-1} \mathrm{~cm}^{-2} \operatorname{arcsec}^{-2}$.

Median values over a $40^{\prime \prime} \times 40^{\prime \prime}$ box.

${ }^{a}$ The west [O III] bright filament outside the SNR's borders.

${ }^{b} 3 \sigma$ upper limit.

recombination zone where the low ionization lines are produced, and it could also explain the $[\mathrm{O} \mathrm{III}] / \mathrm{H} \alpha$ ratio variations seen in the long-slit spectra.

An interstellar extinction c between (see Table 3) 1.17 $( \pm 0.05)$ and $1.68( \pm 0.05)$ or an $A_{\mathrm{V}}$ between $2.51( \pm 0.11)$ and $3.60( \pm 0.10)$ were measured, respectively. We have also determined the electron density by measuring the density sensitive line ratio of $[\mathrm{S} \mathrm{II}] \lambda \lambda 6716 / 6731$. The densities we measure are below $250 \mathrm{~cm}^{-3}$. Assuming that the temperature is close to $10^{4} \mathrm{~K}$, it is possible to estimate basic SNR parameters. The remnant under investigation is one of the least studied remnants and thus, the current stage of its evolution is unknown. Assuming that the remnant is still in the adiabatic phase of its evolution, the preshock cloud density $n_{\mathrm{c}}$ can be measured by using the relationship (Dopita 1979)

$n_{[\mathrm{SII}]} \simeq 45 n_{\mathrm{c}} V_{\mathrm{s}}^{2} \mathrm{~cm}^{-3}$, where $n_{[\mathrm{SII}]}$ is the electron density derived from the sulfur line ratio, and $V_{\mathrm{s}}$ the shock velocity into the clouds in units of $100 \mathrm{~km} \mathrm{~s}^{-1}$. Furthermore, the blast wave energy can be expressed in terms of the cloud parameters by using the equation given by McKee \& Cowie (1975):

$E_{51}=2 \times 10^{-5} \beta^{-1} n_{\mathrm{c}} V_{\mathrm{s}}^{2} r_{\mathrm{s}}^{3} \mathrm{erg}$.

The factor $\beta$ is approximately equal to 1 at the blast wave shock, $E_{51}$ the explosion energy in units of $10^{51} \mathrm{erg}$, and $r_{\mathrm{s}}$ the radius of the remnant in pc. By using the upper limit on the electron density of $250 \mathrm{~cm}^{-3}$, which was derived from our spectra, we obtain from Eq. (1) that $n_{\mathrm{c}} V_{\mathrm{s}}^{2}<5$.6. Then Eq. (2) becomes $E_{51}<$ $9 \times 10^{-3} D_{1 \mathrm{kpc}}^{3}$, where $D_{1 \mathrm{kpc}}$ the distance to the remnant in units of $1 \mathrm{kpc}$.

Estimated values of $N_{\mathrm{H}} \sim 6.6 \times 10^{21} \mathrm{~cm}^{-2}$ and $N_{\mathrm{H}} \sim$ $8.3 \times 10^{21} \mathrm{~cm}^{-2}$ are given by Dickey \& Lockman (1990) and Kalberla et al. (2005) respectively, for the column density in the direction of G 15.1-1.6. Using the relation of Ryter et al. (1975), we obtain an $N_{\mathrm{H}}$ of $5.5 \times 10^{21} \mathrm{~cm}^{-2}$ and $7.9 \times 10^{21} \mathrm{~cm}^{-2}$ for the minimum and maximum c values calculated from our spectra, respectively. Both values are consistent with the estimated galactic $N_{\mathrm{H}}$ considering the uncertainties involved. Since, there are no other measurements of the interstellar density $n_{0}$, values of 0.1 and 1.0 will be examined. Following the result of Eq. (2) and assuming the typical value of 1 for the supernova explosion energy $\left(E_{51}\right)$, we find that the remnant may lie at a distance greater than $2.2 \mathrm{kpc}$. Then, the lower interstellar density of $\sim 0.1 \mathrm{~cm}^{-3} \mathrm{sug}$ gests that the column density is greater than $1.4 \times 10^{21} \mathrm{~cm}^{-2}$, while for $n_{0} \approx 1 \mathrm{~cm}^{-3}$ it becomes greater than $1.4 \times 10^{22} \mathrm{~cm}^{-2}$. Combining the previous results and assuming that the column 
Table 3. Relative line fluxes.

\begin{tabular}{|c|c|c|c|c|c|c|c|c|c|}
\hline \multirow[b]{2}{*}{ Line $(\AA)$} & \multicolumn{3}{|c|}{ Area BI } & \multicolumn{3}{|c|}{ Area BII } & \multicolumn{3}{|c|}{ Area E } \\
\hline & $F^{a}$ & $I^{b}$ & $S / N^{c}$ & $F$ & $I$ & $S / N$ & $F$ & $I$ & $\mathrm{~S} / \mathrm{N}$ \\
\hline $\mathrm{H} \beta 4861$ & 9 & 35 & $(27)$ & 11 & 35 & (11) & 13 & 35 & $(25)$ \\
\hline [O III] 4959 & 6 & 20 & (20) & 11 & 31 & (12) & 7 & 18 & (17) \\
\hline [O III] 5007 & 7 & 29 & (33) & 14 & 39 & (18) & 18 & 43 & (39) \\
\hline [O I $] 6300$ & 4 & 5 & (35) & 6 & 7 & (14) & 6 & 7 & (33) \\
\hline$[\mathrm{N}$ II $] 6548$ & 26 & 26 & (160) & 20 & 20 & (44) & 16 & 15 & (74) \\
\hline $\mathrm{H} \alpha 6563$ & 100 & 100 & (441) & 100 & 100 & (162) & 100 & 100 & (276) \\
\hline [N II] 6583 & 81 & 79 & (373) & 63 & 62 & (109) & 47 & 47 & (163) \\
\hline [S II] 6716 & 30 & 27 & (161) & 28 & 26 & (52) & 19 & 18 & $(81)$ \\
\hline [S II] 6731 & 24 & 21 & (129) & 22 & 20 & (41) & 14 & 13 & $(62)$ \\
\hline Absolute $\mathrm{H} \alpha$ flux $^{\mathrm{d}}$ & \multicolumn{3}{|c|}{3} & \multicolumn{3}{|c|}{2} & \multicolumn{3}{|c|}{7} \\
\hline$[\mathrm{S} \mathrm{II}] / \mathrm{H} \alpha$ & \multicolumn{3}{|c|}{$0.50 \pm 0.03$} & \multicolumn{3}{|c|}{$0.48 \pm 0.07$} & \multicolumn{3}{|c|}{$0.33 \pm 0.02$} \\
\hline$F(6716) / F(6731)$ & \multicolumn{3}{|c|}{$1.29 \pm 0.10$} & \multicolumn{3}{|c|}{$1.32 \pm 0.25$} & \multicolumn{3}{|c|}{$1.38 \pm 0.12$} \\
\hline$[\mathrm{N} \mathrm{II}] / \mathrm{H} \alpha$ & \multirow{2}{*}{\multicolumn{3}{|c|}{$\begin{array}{l}1.07 \pm 0.02 \\
1.40 \pm 0.09\end{array}$}} & \multicolumn{3}{|c|}{$0.83 \pm 0.04$} & \multicolumn{3}{|c|}{$0.63 \pm 0.01$} \\
\hline$[\mathrm{O} \mathrm{III}] / \mathrm{H} \beta$ & & & & \multicolumn{3}{|c|}{$2.00 \pm 0.30$} & \multicolumn{3}{|c|}{$1.73 \pm 0.12$} \\
\hline$c(\mathrm{H} \beta)$ & \multicolumn{3}{|c|}{$1.68 \pm 0.05$} & \multirow{2}{*}{\multicolumn{3}{|c|}{$0.95 \pm 0.08$}} & \multirow{2}{*}{\multicolumn{3}{|c|}{$\begin{array}{l}1.17 \pm 0.05 \\
0.81 \pm 0.03\end{array}$}} \\
\hline$E_{B-V}$ & \multicolumn{3}{|c|}{$1.16 \pm 0.03$} & & & & & & \\
\hline
\end{tabular}

${ }^{a}$ Observed fluxes normalized to $F(\mathrm{H} \alpha)=100$ and uncorrected for interstellar extinction.

${ }^{b}$ Intrinsic fluxes normalized to $F(\mathrm{H} \alpha)=100$ and corrected for interstellar extinction.

${ }^{c}$ Numbers in parentheses represent the signal-to-noise ratio of the quoted fluxes.

${ }^{d}$ In units of $10^{-16} \mathrm{erg} \mathrm{s}^{-1} \mathrm{~cm}^{-2} \operatorname{arcsec}{ }^{-2}$.

Listed fluxes are a signal-to-noise weighted average of two fluxes for areas BI and BII.

The emission line ratios $[\mathrm{S} \mathrm{II}] / \mathrm{H} \alpha, F(6716) / F(6731)$ and $[\mathrm{O} \mathrm{III}] / \mathrm{H} \beta$ are calculated using the values corrected for interstellar extinction.

The errors of the emission line ratios, $c(\mathrm{H} \beta)$ and $E_{B-V}$, are calculated through standard error propagation.

density is found in the range of $5-8 \times 10^{21} \mathrm{~cm}^{-2}$, then the lower interstellar density seems to be more probable. However, since neither the distance nor the interstellar medium density are accurately known, we cannot confidently determine the current stage of evolution of G 15.1-1.6.

\section{Conclusions}

The faint supernova remnant $\mathrm{G} 15.1-1.6$ was observed for the first time in major optical emission lines. The images show filamentary and diffuse emission structures. The bright filaments are correlated very well with the remnant's radio emission at $4850 \mathrm{MHz}$ suggesting their association, while correlation evidence is also shown with the IRAS $60 \mu \mathrm{m}$ map. The fluxcalibrated images and the long-slit spectra indicate that the emission arises from shock-heated gas. Finally, an upper limit for the electron density of $250 \mathrm{~cm}^{-3}$ and a lower limit for the distance of $2.2 \mathrm{kpc}$ are calculated.

Acknowledgements. J.A. acknowledges funding by the European Union and the Greek Ministry of Development in the framework of the program "Promotion of Excellence in Research Institutes (2nd Part)". Skinakas Observatory is a collaborative project of the University of Crete, the Foundation for Research and Technology-Hellas, and the Max-Planck-Institut für Extraterrestrische Physik.

\section{References}

Blair, W. P., Sankrit, R., \& Raymond, J. C. 2005, AJ, 129, 2268

Boumis, P., Mavromatakis, F., \& Paleologou, E. V. 2002, A\&A, 385, 1042
Boumis, P., Mavromatakis, F., Xilouris, E. M., et al. 2005, A\&A, 443, 175

Cox, D. P., \& Raymond, J. C. 1985, ApJ, 298, 651

Dickey, J. M., \& Lockman, F. J. 1990, ARA\&A, 28, 215

Dopita, M. A. 1979, ApJS, 40, 455

Fesen, R. A., Blair, W. P., \& Kirshner, R. P. 1985, ApJ, 292, 29

Green, D. A. 2006, A Catalog of Galactic Supernova Remnants (2006 April version), Mullard Radio Astronomy Observatory Cambridge

Hamuy, M., Walker, A. R., \& Suntzeff, N. B. 1992, PASP, 104, 533

Hartigan, P., Raymond, J., \& Hartmann, L. 1987, ApJ, 316, 323

Kalberla, P. M. W., Burton, W. B., Hartmann, D., et al. 2005, A\&A, 440, 775

Lasker, B. M., Russel, J. N., \& Jenkner, H. 1999, in the HST Guide Star Catalog, version 1.1-ACT, The Association of Universities for Research in Astronomy, Inc.

Lockman, F. J. 1989, ApJS, 71, 469

Mavromatakis, F., Boumis, P., Papamastorakis, J., \& Ventura, J. 2002, A\&A, 388,355

Mavromatakis, F., Boumis, P., Xilouris, E. M., et al. 2005, A\&A, 435, 141

McKee, C. F., \& Cowie, L. L. 1975, ApJ, 195, 715

Osterbrock, D. E., \& Ferland, G. J. 2006, Astrophysics of gaseous nebulae and AGN, ed. University Science Books, US

Raymond, J. C., Blair, W. P., Fesen, R. A., \& Gull, T. R. 1983, ApJ, 275, 636

Raymond, J. C., Hester, J. J., Cox, D., et al. 1988, ApJ, 324, 869

Reich, W., Fürst, E., Reich, P., \& Junkes, N. 1988, in Supernova Remnants and the Interstellar Medium, ed. R. S. Rogerand, \& T. L. Landecker (Cambridge Univ. Press), IAU Colloq., 101, 293

Reich, W., Fürst E., Reich, P., \& Reif, K. 1990, A\&AS, 85, 633

Ryter, C., Cesarsky, C. J., \& Audouze, J. 1975, ApJ, 198, 103

Seward, F. D., Dame, T. M., Fesen, R. A., \& Aschenbach, B. 1995, ApJ, 449, 681

Shaw, R. A., \& Dufour, R. J. 1995, PASP, 107, 896 